\title{
Perfect Solvent- and Catalyst-Free Syntheses of Imine Derivatives Using the Pressure Reduction Technique
}

\author{
Shoko Suzuki', Hiroyuki Ito², Shinji Ishizuka², Risehiro Nonaka², Motoyoshi Noike², \\ Takeshi Kodama' ${ }^{2}$ Kenji Funaki ${ }^{2}$, Mizuho Taguchi' ${ }^{3}$, Taisei Kagaya ${ }^{4}$, Sayaka Sato ${ }^{4}$, \\ Guillaume Redler ${ }^{5}$, Yasuo Yokoyama ${ }^{2 *}$ \\ ${ }^{1}$ Faculty of Education, Kyoto University of Education, Kyoto, Japan \\ ${ }^{2}$ Department of Chemical and Biological Engineering, National Institute of Technology, Akita College, Akita, Japan \\ ${ }^{3}$ Environment Systems Engineering Course, National Institute of Technology, Akita College, Akita, Japan \\ ${ }^{4}$ Department of Applied Chemistry, National Institute of Technology, Akita College, Akita, Japan \\ ${ }^{5}$ Science et Génie des Matériaux, IUT Blois, Blois, France \\ Email: *yokoyama@akita-nct.ac.jp
}

How to cite this paper: Suzuki, S., Ito, H., Ishizuka, S., Nonaka, R., Noike, M., Kodama, T., Funaki, K., Taguchi, M., Kagaya, T., Sato, S., Redler, G. and Yokoyama, Y. (2019) Perfect Solvent- and Catalyst-Free Syntheses of Imine Derivatives Using the Pressure Reduction Technique. Green and Sustainable Chemistry, 9, 105-118. https://doi.org/10.4236/gsc.2019.94008

Received: August 21, 2019

Accepted: October 9, 2019

Published: October 12, 2019

Copyright $\odot 2019$ by author(s) and Scientific Research Publishing Inc. This work is licensed under the Creative Commons Attribution International License (CC BY 4.0).

http://creativecommons.org/licenses/by/4.0/

\section{(c) (i) Open Access}

\begin{abstract}
In the field of organic syntheses, the development of environmentally friendly methods based on the concept of green chemistry has been always required. In response to this requirement, we reported solvent- and catalyst-free syntheses of imines using the pressure reduction technique as a key technology. We found that this reaction proceeded very rapidly in the initial stage, but its rate decreased with the passage of time. It was also found that the reaction of benzaldehyde with aniline had a specificity that the phase transition occurred. In this method, the desired imines could be obtained in good to excellent yields, but target compounds had to be given by purifications using organic solvents. Therefore, we tried to develop the perfect synthetic method of imine derivatives without organic or inorganic solvents. We selected two methods and took them into this investigation. One was exactly mixing (1:1, substance ratio) aldehydes and amines and the other was employing lower pressure ( $>0.1 \mathrm{mmHg}$, previous method: $1.0 \mathrm{mmHg}$ ) at the pressure reducing technique. When this improved synthetic method was performed, it was revealed that pure target imines were obtained in excellent yields without any purification.
\end{abstract}

\section{Keywords}

Solvent-Free, Catalyst-Free, Neat Reaction, Pressure Reduction Technique, Imine 


\section{Introduction}

From the viewpoint of green chemistry, it was considered that using organic solvents in organic syntheses aimed at formation convenient compounds was not environmentally friendly, in recent years. Several attempts have been made to avoid using organic solvents in organic synthetic methods. Among them, the method attracting attention was to use water instead of an organic solvent [1] [2] [3] [4]. These investigations were very interesting because water was indispensable to the natural environment and was unlikely to cause environmental destruction. Since water was more inexpensive than organic solvents, the cost of the synthetic reaction could be reduced. Furthermore, it was possible to develop a completely different reaction field when organic reactions proceeded in water, and further development was expected in the future. On the other hand, a solvent-free reaction had also been recognized as a suitable reaction for green chemistry [5] [6] [7] [8]. Therefore, various solvent-free reaction systems had been developed [9] [10] [11]. In the last year, we reported that efficient solvent- and catalyst-free syntheses of imine derivatives apply the pressure reduction technique [12]. And more, other researchers also reported that reducing pressure technology was effective for syntheses of some imines [13]. In our previous reaction system, it was found that target imines could be obtained in good yields by removing water under reduced pressure conditions. We also found that this reaction proceeded rapidly in the initial stage, but its rate decreased with the passage of time. Although this reaction system gave target products in good yields, the purification of the target product was required, such as the column chromatography using hexane/ether combination or the recrystallization using dichloromethane/hexane combination. Thus, our previous system was not a complete solvent-free synthesis system. Therefore, we tried two methods in order to improve this point. One was exactly mixing (1:1, substance ratio) aldehydes and amines and the other was employing lower pressure $(>0.1 \mathrm{mmHg}$, previous method: $1.0 \mathrm{mmHg}$ ) at the pressure reducing technique to remove water and unreacted materials perfectly. Actually, it was found that pure target imines were obtained in excellent yields without any purification using organic solvents, when we tried improved reaction methods (Scheme).

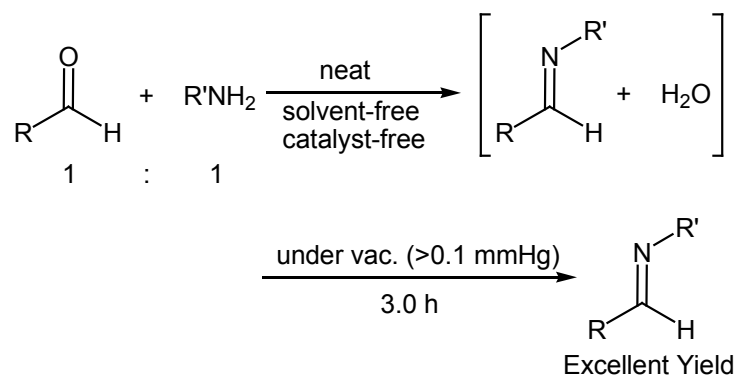

Scheme. Solvent- and Catalyst-free Syntheses of Imines

In this paper, we will report the details of the improved reaction method. 


\section{Experimental}

\subsection{Chemicals and Instruments}

Standard bench top techniques were employed for handling air-sensitive reagent. Liquid aromatic aldehydes and all amines were distilled under argon before use. $p$-Bromobenzaldehyde and $p$-chlorobenzaldehyde were purified by recrystallization before use. All reactions were carried out under nitrogen atmospheres. All yields of target compounds were isolated yields. ULVAC G-50DA (ULVAC KIKO Inc.) was used for carrying out this reducing pressure operation. IR spectra were recorded on an FT/IR-610 (JASCO) spectrophotometer. ${ }^{1} \mathrm{H}-\mathrm{NMR},{ }^{13} \mathrm{C}-\mathrm{NMR}$ and ${ }^{19} \mathrm{~F}-\mathrm{NMR}$ spectra were measured on Bruker BioSpin AVANCE III 400 Nanobay spectrometer at 400.1, 100.6 and $376.5 \mathrm{MHz}$, respectively. Chemical shifts were given in ppm relative to TMS $\left({ }^{1} \mathrm{H}\right.$ and $\left.{ }^{13} \mathrm{C}\right)$ or $\mathrm{CFCl}_{3}$ $\left({ }^{19} \mathrm{~F}\right)$.

\subsection{Typical Experimental Procedure 1 (Reaction of $p$-Tolualdehyde with $o$-Toluidine)}

To a stirring $p$-tolualdehyde $(1.20 \mathrm{~g}, 10.0 \mathrm{mmol})$ was added dropwise $o$-toluidine $(1.07 \mathrm{~g}, 10.0 \mathrm{mmol})$ at $25^{\circ} \mathrm{C}$ After 1.5 hours, the reaction system was connected to a vacuum pump, the pressure was reduced to $>0.1 \mathrm{mmHg}(13.3 \mathrm{~Pa})$ and stirred for 3.0 hours to give the desired pure compound (Compound 1) as a pale yellow clear oil in $99 \%$ yield (2.06 g) without any purification. All physical properties of this product were completely consistent with literature values [14] [15] or physical data of the commercially available compound.: IR (neat): 495, 720, 756, 815, 882, 1111, 1172, 1485, 1572, 1594, 1608, 1627, 2856, 2921, 3021, 3060 $\mathrm{cm}^{-1} ;{ }^{1} \mathrm{H}-\mathrm{NMR}\left(\mathrm{CDCl}_{3}\right) \delta(\mathrm{ppm}): 2.33\left(\mathrm{~s}, 3 \mathrm{H}, \mathrm{H}^{1}\right), 2.40\left(\mathrm{~s}, 3 \mathrm{H}, \mathrm{H}^{2}\right), 6.89(\mathrm{~d}, J=$ $\left.7.80 \mathrm{~Hz}, 1 \mathrm{H}, \mathrm{H}^{3}\right), 7.03-7.11\left(\mathrm{~m}, 1 \mathrm{H}, \mathrm{H}^{4}\right), 7.16-7.22\left(\mathrm{~m}, 2 \mathrm{H}, \mathrm{H}^{5}\right.$ and $\left.\mathrm{H}^{6}\right), 7.26(\mathrm{~d}$, $\left.J=7.92 \mathrm{~Hz}, 2 \mathrm{H}, \mathrm{H}^{7}\right), 7.79\left(\mathrm{~d}, J=7.92 \mathrm{~Hz}, 2 \mathrm{H}, \mathrm{H}^{8}\right), 8.30\left(\mathrm{~s}, 1 \mathrm{H}, \mathrm{H}^{9}\right) ;{ }^{13} \mathrm{C}-\mathrm{NMR}$ $\left(\mathrm{CDCl}_{3}\right) \delta(\mathrm{ppm}): 17.69\left(\mathrm{C}^{1}\right), 21.46\left(\mathrm{C}^{2}\right), 117.56\left(\mathrm{C}^{3}\right), 125.27\left(\mathrm{C}^{4}\right), 126.52\left(\mathrm{C}^{5}\right)$, $128.55\left(C^{6}\right), 129.30\left(C^{7}\right), 130.02\left(C^{8}\right), 131.61\left(C^{9}\right), 133.74\left(C^{10}\right), 141.50\left(C^{11}\right)$, $151.14\left(\mathrm{C}^{12}\right), 159.23\left(\mathrm{C}^{13}\right)$.

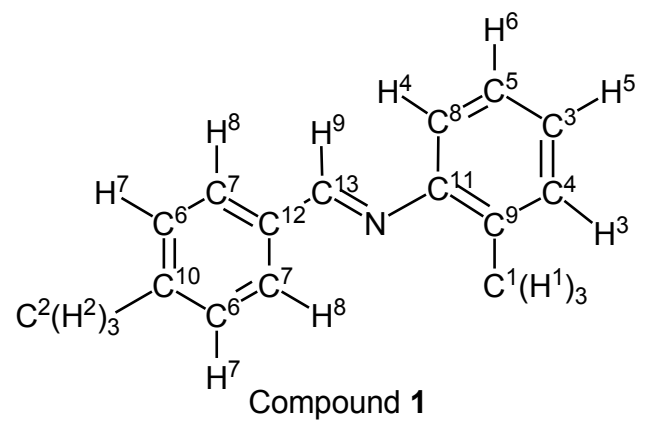

p-Tolualdehyde $(1.20 \mathrm{~g}, 10.0 \mathrm{mmol})$ reacted with $m$-toluidine (1.07 $\mathrm{g}, 10$ $\mathrm{mmol}$ ) to give the corresponding pure compound (Compound 2) in 99\% yield $(2.06 \mathrm{~g})$ as a colorless crystal in the same manner of procedure 1. All physical 
properties of this product were completely consistent with literature values [16] or physical data of the commercially available compound.: IR (neat): 479, 694, 774, 792, 816, 930, 1148, 1173, 1307, 1483, 1573, 1599, 1627, 2731, 2863, 2919, $3024 \mathrm{~cm}^{-1}$; ${ }^{1} \mathrm{H}-\mathrm{NMR}\left(\mathrm{CDCl}_{3}\right) \delta(\mathrm{ppm}): 2.39\left(\mathrm{~s}, 3 \mathrm{H}, \mathrm{H}^{1}\right), 2.42\left(\mathrm{~s}, 3 \mathrm{H}, \mathrm{H}^{2}\right), 7.00-$ $7.05\left(\mathrm{~m}, 3 \mathrm{H}, \mathrm{H}^{3}, \mathrm{H}^{4}\right.$ and $\left.\mathrm{H}^{5}\right), 7.24-7.30\left(\mathrm{~m}, 1 \mathrm{H}, \mathrm{H}^{6}\right), 7.28(\mathrm{~d}, J=8.12 \mathrm{~Hz}, 2 \mathrm{H}$, $\left.\mathrm{H}^{7}\right), 7.79\left(\mathrm{~d}, J=8.12 \mathrm{~Hz}, 2 \mathrm{H}, \mathrm{H}^{8}\right), 8.41\left(\mathrm{~s}, 1 \mathrm{H}, \mathrm{H}^{9}\right) ;{ }^{13} \mathrm{C}-\mathrm{NMR}\left(\mathrm{CDCl}_{3}\right) \delta(\mathrm{ppm})$ : $21.44\left(\mathrm{C}^{1}\right), 21.66\left(\mathrm{C}^{2}\right), 117.86\left(\mathrm{C}^{3}\right), 121.66\left(\mathrm{C}^{4}\right), 126.54\left(\mathrm{C}^{5}\right), 128.79\left(\mathrm{C}^{6}\right), 128.96$ $\left(C^{7}\right), 129.52\left(C^{8}\right), 133.71\left(C^{9}\right), 138.96\left(C^{10}\right), 141.81\left(C^{11}\right), 152.25\left(C^{12}\right), 160.19$ $\left(\mathrm{C}^{13}\right)$.

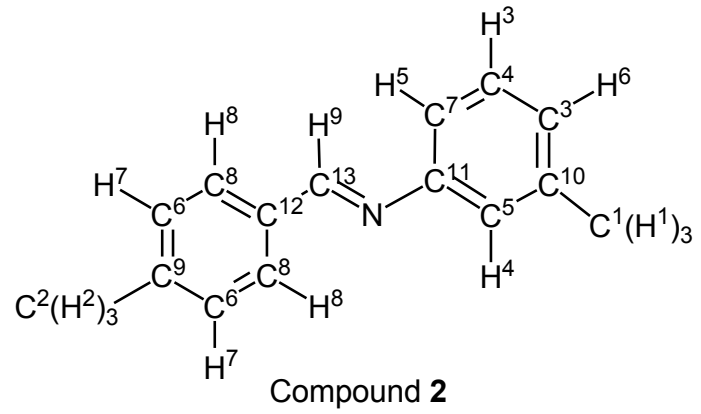

p-Tolualdehyde $(1.20 \mathrm{~g}, 10.0 \mathrm{mmol})$ reacted with $p$-toluidine $(1.07 \mathrm{~g}, 10$ $\mathrm{mmol}$ ) to give the corresponding pure compound (Compound 3 ) in $98 \%$ yield $(2.05 \mathrm{~g})$ as a colorless crystal in the same manner of procedure 1 . All physical properties of this product were completely consistent with literature values [17] [18] [19] or physical data of the commercially available compound.: IR (neat): 467, 539, 718, 823, 885, 977, 1108, 1170, 1504, 1568, 1605, 1624, 2859, 2915, 3026 $\mathrm{cm}^{-1} ;{ }^{1} \mathrm{H}-\mathrm{NMR}\left(\mathrm{CDCl}_{3}\right) \delta(\mathrm{ppm}): 2.36\left(\mathrm{~s}, 3 \mathrm{H}, \mathrm{H}^{1}\right), 2.41\left(\mathrm{~s}, 3 \mathrm{H}, \mathrm{H}^{2}\right), 7.13(\mathrm{~d}, J=$ $\left.8.20 \mathrm{~Hz}, 2 \mathrm{H}, \mathrm{H}^{3}\right), 7.19\left(\mathrm{~d}, J=8.20 \mathrm{~Hz}, 2 \mathrm{H}, \mathrm{H}^{4}\right), 7.27\left(\mathrm{~d}, J=7.96 \mathrm{~Hz}, 2 \mathrm{H}, \mathrm{H}^{5}\right), 7.78$ $\left(\mathrm{d}, J=7.96 \mathrm{~Hz}, 2 \mathrm{H}, \mathrm{H}^{6}\right), 8.42\left(\mathrm{~s}, 1 \mathrm{H}, \mathrm{H}^{7}\right) ;{ }^{13} \mathrm{C}-\mathrm{NMR}\left(\mathrm{CDCl}_{3}\right) \delta(\mathrm{ppm}): 21.04\left(\mathrm{C}^{1}\right)$, $21.66\left(C^{2}\right), 120.83\left(C^{3}\right), 128.73\left(C^{4}\right), 129.52\left(C^{5}\right), 129.76\left(C^{6}\right), 133.79\left(C^{7}\right), 135.60$ $\left(C^{8}\right), 141.69\left(C^{9}\right), 149.64\left(C^{10}\right), 159.65\left(C^{11}\right)$.

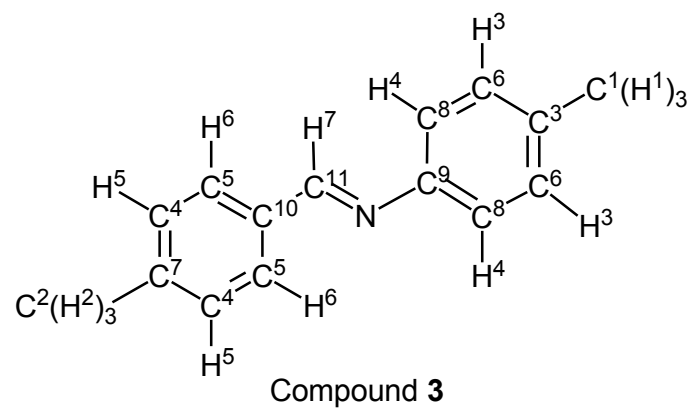

$p$-Anisaldehyde (1.36 g, $10.0 \mathrm{mmol})$ reacted with $o$-toluidine $(1.07 \mathrm{~g}, 10$ $\mathrm{mmol}$ ) to give the corresponding pure compound (Compound 4) in $99 \%$ yield $(2.24 \mathrm{~g})$ as a colorless crystal in the same manner of procedure 1. All physical properties of this product were completely consistent with literature values [20] 
[21] [22] or physical data of the commercially available compound.: IR (neat): 450, 517, 724, 757, 832, 883, 1031, 1107, 1164, 1253, 1309, 1511, 1574, 1605, 2332, 2559, 2838, 2910, 2934, 2955, 3009, $3065 \mathrm{~cm}^{-1}$; ${ }^{1} \mathrm{H}-\mathrm{NMR}\left(\mathrm{CDCl}_{3}\right) \delta$ (ppm): $2.35\left(\mathrm{~s}, 3 \mathrm{H}, \mathrm{H}^{1}\right), 3.86\left(\mathrm{~s}, 3 \mathrm{H}, \mathrm{H}^{2}\right), 6.89-6.91\left(\mathrm{~m}, 1 \mathrm{H}, \mathrm{H}^{3}\right), 6.98(\mathrm{~d}, J=8.72 \mathrm{~Hz}$, $\left.2 \mathrm{H}, \mathrm{H}^{4}\right), 7.08-7.12\left(\mathrm{~m}, 1 \mathrm{H}, \mathrm{H}^{5}\right), 7.18-7.29\left(\mathrm{~m}, 2 \mathrm{H}, \mathrm{H}^{6}\right.$ and $\left.\mathrm{H}^{7}\right), 7.85(\mathrm{~d}, J=8.72$ $\left.\mathrm{Hz}, 2 \mathrm{H}, \mathrm{H}^{8}\right), 8.28\left(\mathrm{~s}, 1 \mathrm{H}, \mathrm{H}^{9}\right) ;{ }^{13} \mathrm{C}-\mathrm{NMR}\left(\mathrm{CDCl}_{3}\right) \delta(\mathrm{ppm}): 17.73\left(\mathrm{C}^{1}\right), 55.26\left(\mathrm{C}^{2}\right)$, $113.96\left(C^{3}\right), 117.61\left(C^{4}\right), 125.11\left(C^{5}\right), 126.53\left(C^{6}\right), 129.37\left(C^{7}\right), 130.02\left(C^{8}\right), 130.22$ $\left(C^{9}\right), 131.60\left(C^{10}\right), 151.24\left(C^{11}\right), 158.56\left(C^{12}\right), 161.94\left(C^{13}\right)$.

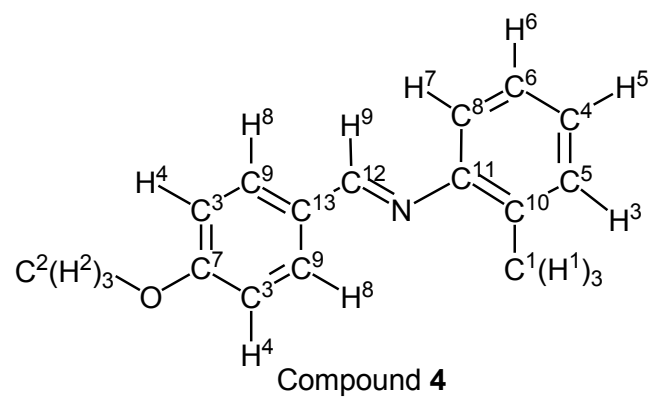

$p$-Anisaldehyde $(1.36 \mathrm{~g}, 10.0 \mathrm{mmol})$ reacted with $m$-toluidine $(1.07 \mathrm{~g}, 10$ $\mathrm{mmol}$ ) to give the corresponding pure compound (Compound 5) in $99 \%$ yield $(2.24 \mathrm{~g})$ as a colorless crystal in the same manner of procedure 1. All physical properties of this product were completely consistent with literature values [21] [23] or physical data of the commercially available compound.: IR (neat): 443, $530,694,792,834,933,1024,1105,1165,1246,1309,1419,1457,1508,1604$, 1621, 2554, 2839, 2910, 2935, $2966 \mathrm{~cm}^{-1} ;{ }^{1} \mathrm{H}-\mathrm{NMR}\left(\mathrm{CDCl}_{3}\right) \delta(\mathrm{ppm}): 2.38(\mathrm{~s}, 3 \mathrm{H}$, $\left.\mathrm{H}^{1}\right), 3.87\left(\mathrm{~s}, 3 \mathrm{H}, \mathrm{H}^{2}\right), 6.95-7.05\left(\mathrm{~m}, 3 \mathrm{H}, \mathrm{H}^{3}, \mathrm{H}^{4}\right.$ and $\left.\mathrm{H}^{5}\right), 6.98(\mathrm{~d}, J=8.72 \mathrm{~Hz}, 2 \mathrm{H}$, $\left.\mathrm{H}^{6}\right), 7.25-7.29\left(\mathrm{~m}, 1 \mathrm{H}, \mathrm{H}^{7}\right), 7.84\left(\mathrm{~d}, J=8.72 \mathrm{~Hz}, 2 \mathrm{H}, \mathrm{H}^{8}\right), 8.39\left(\mathrm{~s}, 1 \mathrm{H}, \mathrm{H}^{9}\right)$; ${ }^{13} \mathrm{C}-\mathrm{NMR}\left(\mathrm{CDCl}_{3}\right) \delta$ (ppm): $21.45\left(\mathrm{C}^{1}\right), 55.45\left(\mathrm{C}^{2}\right), 114.18\left(\mathrm{C}^{3}\right), 117.87\left(\mathrm{C}^{4}\right)$, $121.66\left(C^{5}\right), 126.36\left(C^{6}\right), 128.96\left(C^{7}\right), 129.32\left(C^{8}\right), 130.49\left(C^{9}\right), 138.94\left(C^{10}\right)$, $152.37\left(\mathrm{C}^{11}\right), 159.54\left(\mathrm{C}^{12}\right), 162.19\left(\mathrm{C}^{13}\right)$.

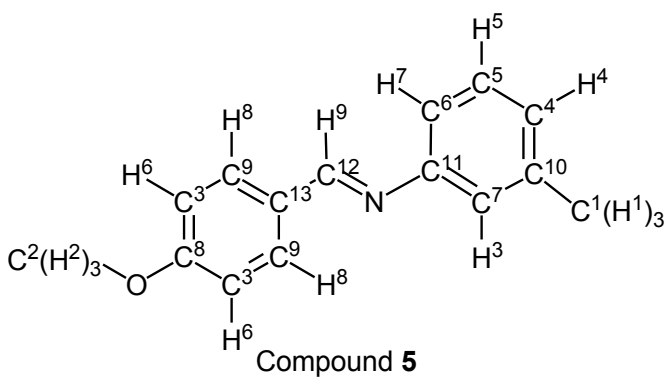

$p$-Anisaldehyde (1.36 g, $10.0 \mathrm{mmol})$ reacted with $p$-toluidine $(1.07 \mathrm{~g}, 10$ $\mathrm{mmol}$ ) to give the corresponding pure compound (Compound 6 ) in $98 \%$ yield $(2.21 \mathrm{~g})$ as a colorless crystal in the same manner of procedure 1 . All physical properties of this product were completely consistent with literature values [18] [24] [25] [26] [27] or physical data of the commercially available compound.: IR 
(neat): 505, 546, 590, 820, 838, 1025, 1105, 1166, 1249, 1420, 1508, 1570, 1604, 2555, 2841, 2876, 2971, $3005 \mathrm{~cm}^{-1} ;{ }^{1} \mathrm{H}-\mathrm{NMR}\left(\mathrm{CDCl}_{3}\right) \delta(\mathrm{ppm}): 2.37\left(\mathrm{~s}, 3 \mathrm{H}, \mathrm{H}^{1}\right)$, $3.87\left(\mathrm{~s}, 3 \mathrm{H}, \mathrm{H}^{2}\right), 6.98\left(\mathrm{~d}, J=8.80 \mathrm{~Hz}, 2 \mathrm{H}, \mathrm{H}^{3}\right), 7.12\left(\mathrm{~d}, J=8.04 \mathrm{~Hz}, 2 \mathrm{H}, \mathrm{H}^{4}\right), 7.17$ $\left(\mathrm{d}, J=8.04 \mathrm{~Hz}, 2 \mathrm{H}, \mathrm{H}^{5}\right), 7.84\left(\mathrm{~d}, J=8.80 \mathrm{~Hz}, 2 \mathrm{H}, \mathrm{H}^{6}\right), 8.39\left(\mathrm{~s}, 1 \mathrm{H}, \mathrm{H}^{7}\right) ;{ }^{13} \mathrm{C}-\mathrm{NMR}$ $\left(\mathrm{CDCl}_{3}\right) \delta(\mathrm{ppm}): 20.99\left(\mathrm{C}^{1}\right), 55.42\left(\mathrm{C}^{2}\right), 114.13\left(\mathrm{C}^{3}\right), 120.76\left(\mathrm{C}^{4}\right), 129.35\left(\mathrm{C}^{5}\right)$, $129.71\left(C^{6}\right), 130.38\left(C^{7}\right), 135.35\left(C^{8}\right), 149.72\left(C^{9}\right), 159.00\left(C^{10}\right), 162.08\left(C^{11}\right)$.

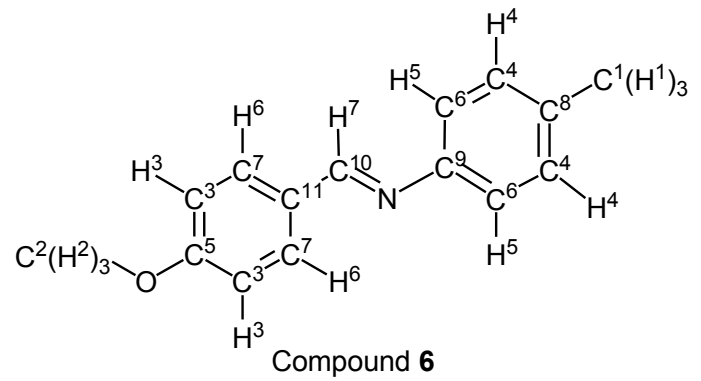

\subsection{Typical Experimental Procedure 2 (Reaction of $p$-Tolualdehyde with Benzylamine)}

To a stirring $p$-tolualdehyde $(1.20 \mathrm{~g}, 10.0 \mathrm{mmol})$ was added dropwise benzylamine $(1.07 \mathrm{~g}, 10.0 \mathrm{mmol})$ at $25^{\circ} \mathrm{C}$. After 2.0 hours, the reaction system was connected to a vacuum pump, the pressure was reduced to $>0.1 \mathrm{mmHg}(13.3 \mathrm{~Pa})$ and stirred for 3.0 hours to give the desired pure compound (Compound 7) as a white solid in $97 \%$ yield ( $2.03 \mathrm{~g}$ ) without any purification. All physical properties of this product were completely consistent with literature values [28] [29] or physical data of the commercially available compound.: IR (neat): 442, 505, 697, 733, 819, 865, 1028, 1173, 1307, 1377, 1453, 1496, 1606, 1648, 2837, 2920, 3027, $3061 \mathrm{~cm}^{-1} ;{ }^{1} \mathrm{H}-\mathrm{NMR}\left(\mathrm{CDCl}_{3}\right) \delta(\mathrm{ppm}): 2.38\left(\mathrm{~s}, 3 \mathrm{H}, \mathrm{H}^{1}\right), 4.80\left(\mathrm{~s}, 2 \mathrm{H}, \mathrm{H}^{2}\right), 7.22(\mathrm{~d}, J$ $\left.=7.96 \mathrm{~Hz}, 2 \mathrm{H}, \mathrm{H}^{3}\right), 7.24-7.28\left(\mathrm{~m}, 1 \mathrm{H}, \mathrm{H}^{4}\right), 7.32-7.36\left(\mathrm{~m}, 4 \mathrm{H}, \mathrm{H}^{5}\right.$ and $\left.\mathrm{H}^{6}\right), 7.67$ $\left(\mathrm{d}, J=7.96 \mathrm{~Hz}, 2 \mathrm{H}, \mathrm{H}^{7}\right), 8.35\left(\mathrm{~s}, 1 \mathrm{H}, \mathrm{H}^{8}\right) ;{ }^{13} \mathrm{C}-\mathrm{NMR}\left(\mathrm{CDCl}_{3}\right) \delta(\mathrm{ppm}): 21.51\left(\mathrm{C}^{1}\right)$, $65.02\left(C^{2}\right), 126.91\left(C^{3}\right), 127.94\left(C^{4}\right), 128.23\left(C^{5}\right), 128.45\left(C^{6}\right), 129.31\left(C^{7}\right), 133.51$ $\left(C^{8}\right), 139.39\left(C^{9}\right), 141.04\left(C^{10}\right), 161.94\left(C^{11}\right)$.

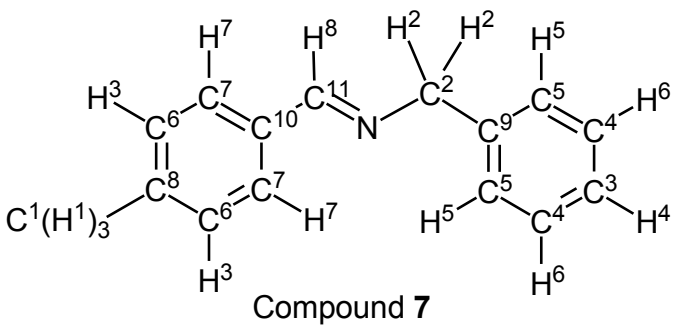

p-Anisaldehyde (1.36 g, $10.0 \mathrm{mmol}$ ) reacted with benzylamine (1.07 g, 10 $\mathrm{mmol}$ ) to give the corresponding pure compound (Compound 8) in 100\% (2.25 g) as a colorless crystal in the same manner of procedure 2. All physical properties of this product were completely consistent with literature values [30] [31] or physical data of the commercially available compound.: IR (neat): 462, 528, 697, 
731, 1033, 1165, 1252, 1308, 1449, 1510, 1604, 1650, 2551, 2813, 2844, 2940, 2969, 2999, $3028 \mathrm{~cm}^{-1}$; ${ }^{1} \mathrm{H}-\mathrm{NMR}\left(\mathrm{CDCl}_{3}\right) \delta(\mathrm{ppm}): 3.84\left(\mathrm{~s}, 3 \mathrm{H}, \mathrm{H}^{1}\right), 4.79(\mathrm{~s}, 2 \mathrm{H}$, $\left.\mathrm{H}^{2}\right), 6.93\left(\mathrm{~d}, J=8.80 \mathrm{~Hz}, 2 \mathrm{H}, \mathrm{H}^{3}\right), 7.23-7.29\left(\mathrm{~m}, 1 \mathrm{H}, \mathrm{H}^{4}\right), 7.32-7.36\left(\mathrm{~m}, 4 \mathrm{H}, \mathrm{H}^{5}\right.$ and $\left.\mathrm{H}^{6}\right), 7.73\left(\mathrm{~d}, J=8.80 \mathrm{~Hz}, 2 \mathrm{H}, \mathrm{H}^{7}\right), 8.32\left(\mathrm{~s}, 1 \mathrm{H}, \mathrm{H}^{8}\right) ;{ }^{13} \mathrm{C}-\mathrm{NMR}\left(\mathrm{CDCl}_{3}\right) \delta$ (ppm): $55.35\left(\mathrm{C}^{1}\right), 64.95\left(\mathrm{C}^{2}\right), 113.96\left(\mathrm{C}^{3}\right), 126.90\left(\mathrm{C}^{4}\right), 127.94\left(\mathrm{C}^{5}\right), 128.45\left(\mathrm{C}^{6}\right)$, $129.06\left(C^{7}\right), 129.83\left(C^{8}\right), 139.52\left(C^{9}\right), 161.35\left(C^{10}\right), 161.67\left(C^{11}\right)$.

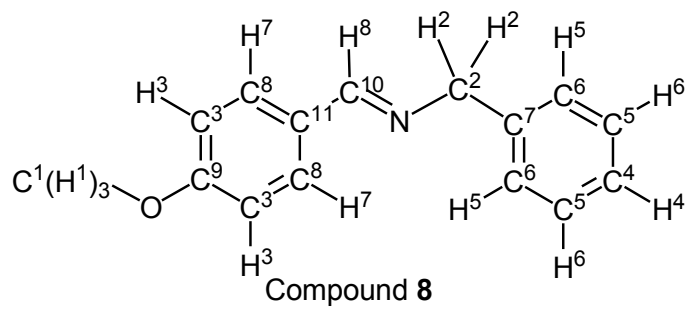

p-Fluorobenzaldehyde $(1.24 \mathrm{~g}, 10.0 \mathrm{mmol})$ reacted with benzylamine $(1.07 \mathrm{~g}$, $10 \mathrm{mmol}$ ) to give the corresponding pure compound (Compound 9) in 99\% $(2.11 \mathrm{~g})$ as a pale yellow clear oil in the same manner of procedure 2. All physical properties of this product were completely consistent with literature values [32] or physical data of the commercially available compound.: IR (neat): 470, 518, $698,734,837,1151,1230,1293,1454,1508,1602,1644,2841,3030,3063 \mathrm{~cm}^{-1}$; ${ }^{1} \mathrm{H}-\mathrm{NMR}\left(\mathrm{CDCl}_{3}\right) \delta(\mathrm{ppm}): 4.81\left(\mathrm{~s}, 2 \mathrm{H}, \mathrm{H}^{1}\right), 7.09\left(\mathrm{t}, J_{\mathrm{HH}}=8.76 \mathrm{~Hz}, J_{\mathrm{HF}}=8.76 \mathrm{~Hz}\right.$, $\left.2 \mathrm{H}, \mathrm{H}^{2}\right), 7.24-7.28\left(\mathrm{~m}, 1 \mathrm{H}, \mathrm{H}^{3}\right), 7.32-7.37\left(\mathrm{~m}, 4 \mathrm{H}, \mathrm{H}^{4}\right.$ and $\left.\mathrm{H}^{5}\right), 7.77\left(\mathrm{dd}, J_{\mathrm{HH}}=\right.$ $\left.8.76 \mathrm{~Hz}, J_{\mathrm{HF}}=5.52 \mathrm{~Hz}, 2 \mathrm{H}, \mathrm{H}^{6}\right), 8.35\left(\mathrm{~s}, 1 \mathrm{H}, \mathrm{H}^{7}\right) ;{ }^{13} \mathrm{C}-\mathrm{NMR}\left(\mathrm{CDCl}_{3}\right) \delta(\mathrm{ppm})$ : $64.94\left(\mathrm{C}^{1}\right), 115.68\left(\mathrm{~d}, J_{\mathrm{CF}}=21.78 \mathrm{~Hz}, \mathrm{C}^{2}\right), 127.04\left(\mathrm{C}^{3}\right), 127.96\left(\mathrm{C}^{4}\right), 128.51\left(\mathrm{C}^{5}\right)$, $130.14\left(\mathrm{~d}, J_{\mathrm{CF}}=8.49 \mathrm{~Hz}, \mathrm{C}^{6}\right), 132.41\left(\mathrm{~d}, J_{\mathrm{CF}}=2.96 \mathrm{~Hz}, \mathrm{C}^{7}\right), 139.14\left(\mathrm{C}^{8}\right), 160.47$ $\left(\mathrm{C}^{9}\right), 164.32\left(\mathrm{~d}, J_{\mathrm{CF}}=250.66 \mathrm{~Hz}, \mathrm{C}^{10}\right) ;{ }^{19} \mathrm{~F}-\mathrm{NMR}\left(\mathrm{CDCl}_{3}\right) \delta(\mathrm{ppm}):-109.86\left(\mathrm{~F}^{1}\right)$.

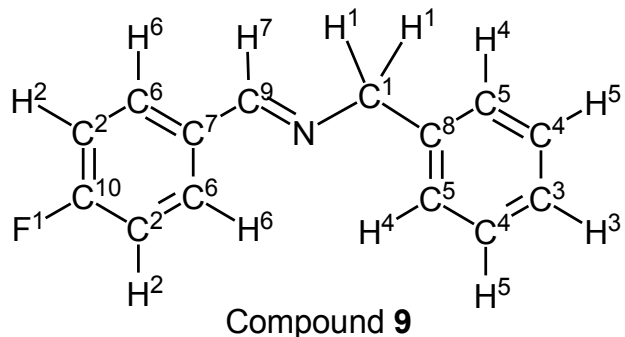

p-Chlorobenzaldehyde $(1.41 \mathrm{~g}, 10.0 \mathrm{mmol})$ reacted with benzylamine $(1.07 \mathrm{~g}$, $10 \mathrm{mmol}$ ) to give the corresponding pure compound (Compound 10) in 99\% $(2.27 \mathrm{~g})$ as a colorless crystal in the same manner of procedure 2. All physical properties of this product were completely consistent with literature values [33] [34] or physical data of the commercially available compound.: IR (neat): 462, 506, 701, 735, 821, 827, 859, 1012, 1044, 1088, 1340, 1371, 1450, 1491, 1593, $1645,2802,2818,2852,2874,3030,3062,3084 \mathrm{~cm}^{-1} ;{ }^{1} \mathrm{H}-\mathrm{NMR}\left(\mathrm{CDCl}_{3}\right) \delta(\mathrm{ppm})$ : $4.81\left(\mathrm{~s}, 2 \mathrm{H}, \mathrm{H}^{1}\right), 7.24-7.29\left(\mathrm{~m}, 1 \mathrm{H}, \mathrm{H}^{2}\right), 7.32-7.36\left(\mathrm{~m}, 4 \mathrm{H}, \mathrm{H}^{3}\right.$ and $\left.\mathrm{H}^{4}\right), 7.38(\mathrm{~d}, J$ $\left.=8.52 \mathrm{~Hz}, 2 \mathrm{H}, \mathrm{H}^{5}\right), 7.71\left(\mathrm{~d}, J=8.52 \mathrm{~Hz}, 2 \mathrm{H}, \mathrm{H}^{6}\right), 8.34\left(\mathrm{~s}, 1 \mathrm{H}, \mathrm{H}^{7}\right) ;{ }^{13} \mathrm{C}-\mathrm{NMR}$ 
$\left(\mathrm{CDCl}_{3}\right) \delta(\mathrm{ppm}): 64.99\left(\mathrm{C}^{1}\right), 127.08\left(\mathrm{C}^{2}\right), 127.97\left(\mathrm{C}^{3}\right), 128.53\left(\mathrm{C}^{4}\right), 128.86\left(\mathrm{C}^{5}\right)$, $129.43\left(C^{6}\right), 134.57\left(C^{7}\right), 136.68\left(C^{8}\right), 138.99\left(C^{9}\right), 160.54\left(C^{10}\right)$.

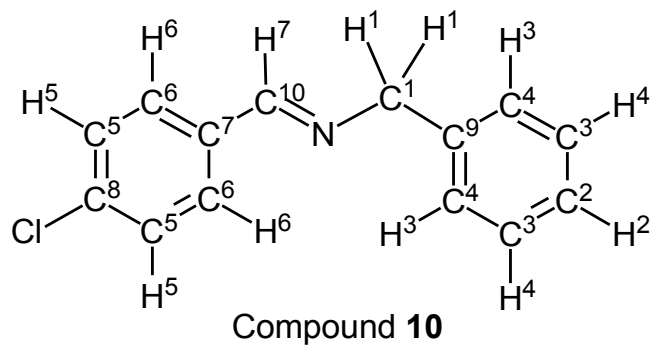

$p$-Bromobenzaldehyde $(1.85 \mathrm{~g}, 10.0 \mathrm{mmol})$ reacted with benzylamine $(1.07 \mathrm{~g}$, $10 \mathrm{mmol}$ ) to give the corresponding pure compound (Compound 11) in $100 \%$ $(2.74 \mathrm{~g})$ as a colorless crystal in the same manner of procedure 2. All physical properties of this product were completely consistent with literature values [35] [36] or physical data of the commercially available compound.: IR (neat): 460, $500,513,698,732,857,1009,1044,1068,1295,1340,1370,1450,1484,1588$, 1644, 2802, 2851, 2874, 3028, 3060, $3082 \mathrm{~cm}^{-1} ;{ }^{1} \mathrm{H}-\mathrm{NMR}\left(\mathrm{CDCl}_{3}\right) \delta$ (ppm): 4.80 $\left(\mathrm{s}, 2 \mathrm{H}, \mathrm{H}^{1}\right), 7.24-7.29\left(\mathrm{~m}, 1 \mathrm{H}, \mathrm{H}^{2}\right), 7.30-7.37\left(\mathrm{~m}, 4 \mathrm{H}, \mathrm{H}^{3}\right.$ and $\left.\mathrm{H}^{4}\right), 7.54(\mathrm{~d}, J=$ $\left.8.48 \mathrm{~Hz}, 2 \mathrm{H}, \mathrm{H}^{5}\right), 7.64\left(\mathrm{~d}, J=8.48 \mathrm{~Hz}, 2 \mathrm{H}, \mathrm{H}^{6}\right), 8.33\left(\mathrm{~s}, 1 \mathrm{H}, \mathrm{H}^{7}\right) ;{ }^{13} \mathrm{C}-\mathrm{NMR}$ $\left(\mathrm{CDCl}_{3}\right) \delta(\mathrm{ppm}): 65.00\left(\mathrm{C}^{1}\right), 125.14\left(\mathrm{C}^{2}\right), 127.08\left(\mathrm{C}^{3}\right), 127.97\left(\mathrm{C}^{4}\right), 128.52\left(\mathrm{C}^{5}\right)$, $129.65\left(C^{6}\right), 131.81\left(C^{7}\right), 134.98\left(C^{8}\right), 138.95\left(C^{9}\right), 160.63\left(C^{10}\right)$.

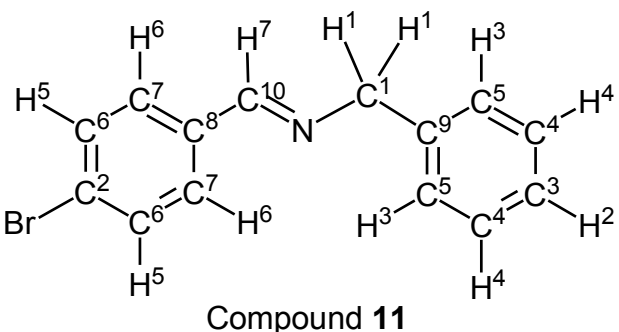

\section{Results and Discussion}

We found that target imines were obtained in excellent yields easily when aldehydes and amines were mixed without a solvent and a catalyst. Some results of reactions of $p$-tolualdehyde or $p$-anisaldehyde with various type toluidines were summarized in Table 1.

$p$-Tolualdehyde reacted with $o$-toluidine for $1.5 \mathrm{~h}$ without any solvent followed by the vacuum operation $(3.0 \mathrm{~h})$ to give the corresponding imine (Compound 1) in $99 \%$ yield (entry 1 ). In this reaction method, any purification, for example, a column chromatography with organic solvents and so on, of the target compound was not needed. The corresponding pure imine (by ${ }^{1} \mathrm{H}-\mathrm{NMR}$ analysis, purity: over 99.5\%) was obtained. This methodology could be applied to the case of $m$-toluidine and $p$-toluidine. When $p$-tolualdehyde and $m$-toluidine were stirred under nitrogen for $1.5 \mathrm{~h}$, the target compound (Com- 
pound 2) was obtained, quantitatively (entry 2 ) after the vacuum operation. We also found that $p$-tolualdehyde reacted with $p$-toluidine, in the same manner, to give the target imine (Compound 3 ) in excellent yield (entry 3 ). Interestingly, an aldehyde that was inert to a nucleophilic reaction could be also used as a starting material for this synthetic method. $p$-Anisaldehyde was inactive for the nucleophilic reaction because it had a strong electron-donating group (methoxy group) at $p$-position. When this type of reaction using $p$-anisaldehyde and $o$-toluidine as starting materials was carried out, the target compound (Compound 4) was obtained in $99 \%$ yield (entry 4 ). $m$-Toluidine and $p$-toluidine also reacted with $p$-anisaldehyde smoothly, and products (Compounds 5 and 6) were given in $99 \%$ yield and $98 \%$ yield (entries 5 and 6). It was reported that Compound 1 was obtained in the highest yield (96\%) when formic acid, water, and ethanol were used as a catalyst and solvents in the previous study [15]. On the other hand, Compound 2 was given in 51\% yield (the highest yield) by iron compounds-promoted C-C bond formation reactions [16]. These facts indicated that our method was more efficient for syntheses of Compounds 1 and 2 because our reactions gave these compounds in higher yields. In the case of the synthesis of Compound 3, it was obtained in $99 \%$ yield by the synthetic reaction using pyridine [17]. In contrast, our reaction gave Compound 3 in a similar yield without any solvent, perfectly. Compounds $\mathbf{4 , 5}$ and $\mathbf{6}$ have already been synthesized by reactions using some solvents and some catalysts in $90 \%$ yield, $90 \%$ yield and 98\% yield, respectively [20] [23] [26]. Our method was more suitable for syntheses of these imines than previous techniques in the viewpoints of yields of target compounds, solvent- and catalyst-free.

Some aldehydes were examined as starting materials for this imine formation and results were shown in Table 2. In this investigation, benzylamine was used as a nucleophile. Although this amine had a milder nucleophilicity than aromatic amines, it had been classified as a strong nucleophile generally.

When aromatic aldehydes having weak or strong electron-donating groups, such as methyl group and methoxy group, were employed as substrates, pure target imines (Compounds 7 and 8 ) were obtained in 97\% yield and 100\% yield (entries 1 and 2). This phenomenon indicated that the ability of electron-donation of a substituent at p-position of the aromatic aldehyde did not affect the yield of an imine at all. Halogenated aromatic aldehydes were also used as starting compounds in this synthetic method. The fluorinated imine derivative (Compound 9) was given in excellent yield by the reaction of $p$-fluorobenzaldehyde and benzylamine (entry 3). Furthermore, $p$-chlorobenzaldehyde and $p$-bromobenzaldehyde could be used as substrates in this reaction system to give target compounds (Compounds 10 and 11) in 99\% yield and 100\% yield (entries 4 and 5). In previous synthetic methods of Compounds 7, 8, 9, 10 and 11, these compounds were obtained in over $90 \%$ yields [28] [30] [32] [33] [36]. Meanwhile, our methods gave these compounds in excellent yields that were comparable to previous methods. Additionally, pure target products could be given without any solvent and any catalyst in our method. 
Table 1. Solvent-and catalyst-free syntheses of imines.

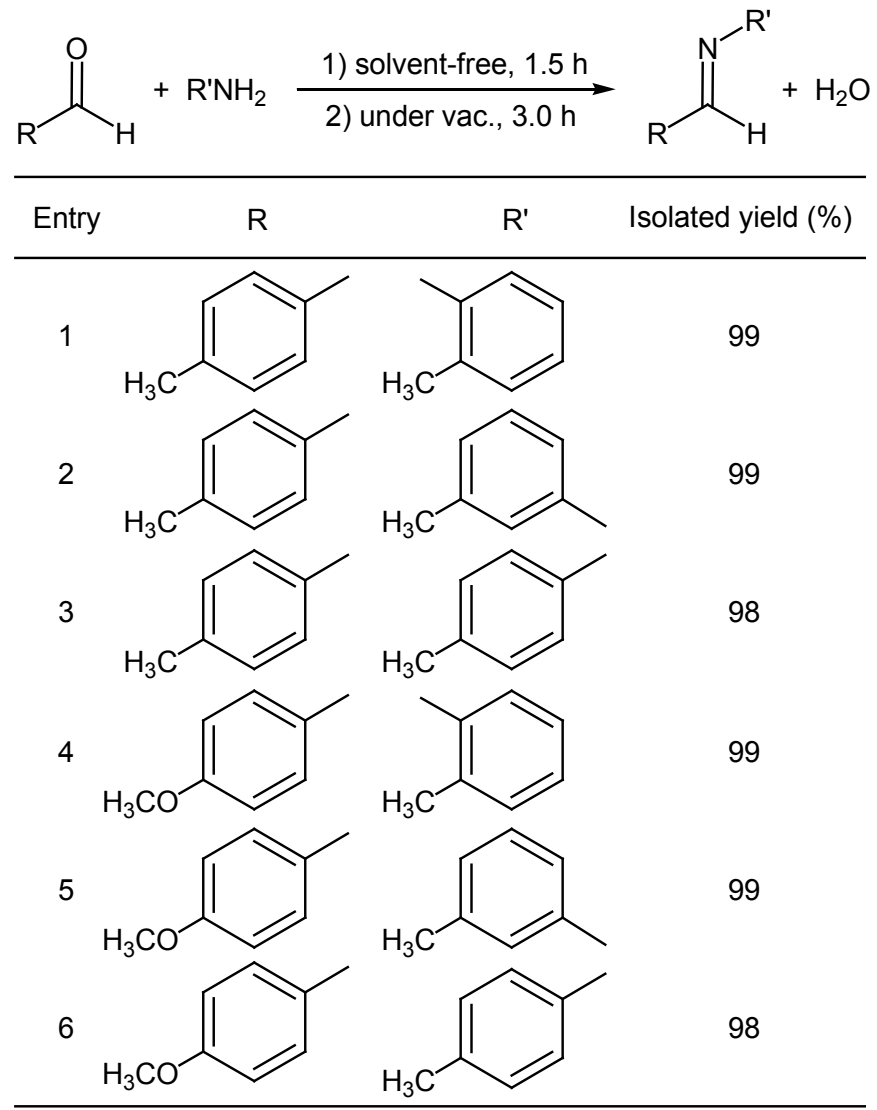

Table 2. Effective syntheses of various benzylated imines.

Isolated yield (\%)


It was revealed that our methodology was superior to the previous one for the synthesis of an imine derivative, because of two reasons that were led from facts in Table 1 and Table 2. The first reason was an excellent yield of a target compound and the second reason was an easy technique under solvent- and catalyst-free conditions.

\section{Conclusion}

In this paper, we reported that perfect solvent- and catalyst-free syntheses of imine derivatives use the pressure reduction technique. This synthetic reaction proceeded under mild conditions, and desired imines were obtained in excellent yields. In this synthetic method, there were two important key-techniques. When aldehydes and amines were mixed exactly 1:1 (substance ratio) and reduced the pressure of the reaction pod under $>0.1 \mathrm{mmHg}$ at the end of this reaction, target imines were obtained, quantitatively. The scope and limitation of this synthetic method are now unknown. Further investigation is in progress.

\section{Conflicts of Interest}

The authors declare no conflicts of interest regarding the publication of this paper.

\section{References}

[1] Bruce, H.L., Nicholas, A.I., James, C.F. and Eric, D.S. (2013) On the Way towards Greener Transition-Metal-Catalyzed Processes as Quantified by E Factors. Angewandte Chemie International Edition, 52, 10952-10958. https://doi.org/10.1002/anie.201302020

[2] Piyatida, K. and Bruce, H.L. (2014) "Nok": A Phytosterol-Based Amphiphile Enabling Transition-Metal-Catalyzed Couplings in Water at Room Temperature. The Journal of Organic Chemistry, 79, 888-900. https://doi.org/10.1021/jo401744b

[3] Tadano, S., Sugimachi, Y., Sumimoto, M., Tsukamoto, S. and Ishikawa, H. (2016) Collective Synthesis and Biological Evaluation of Tryptophan-Based Dimeric Diketopiperazine Alkaloids. Chemistry-A European Journal, 22, 1277-1291. https://doi.org/10.1002/chem.201504560

[4] Kitanosono, T., Masuda, K., Xu, P. and Kobayashi, S. (2018) Catalytic Organic Reactions in Water toward Sustainable Society. Chemical Reviews, 118, 679-746. https://doi.org/10.1021/acs.chemrev.7b00417

[5] Wang, G.-W. (2013) Mechanochemical Organic Synthesis. Chemical Society Reviews, 42, 7668-7700. https://doi.org/10.1039/c3cs35526h

[6] Hernandez, J.G., Avila-Ortiz, C.G. and Juaristi, E. (2014) Useful Chemical Activation Alternatives in Solvent-Free Organic Reactions. In: Knochel, P. and Molander, G.A., Eds., Comprehensive Organic Synthesis, 2nd Edition, Vol. 9, Elsevier B.V., Amsterdam, 287-314. https://doi.org/10.1016/B978-0-08-097742-3.00935-6

[7] Perin, G., Alves, D., Jacob, R.G., Barcellos, A.M., Soares, L.K. and Lenardao, E.J. (2016) Synthesis of Organochalcogen Compounds Using Non-Conventional Reaction Media. ChemistrySelect, 1, 205-258. https://doi.org/10.1002/slct.201500031

[8] Do, J.-L. and Friscic, T. (2017) Chemistry 2.0: Developing a New, Solvent-Free System of Chemical Synthesis Based on Mechanochemistry. Synlett, 28, 2066-2092. 
https://doi.org/10.1055/s-0036-1590854

[9] Ramamurthy, V. and Sivaguru, J. (2016) Supramolecular Photochemistry as a Potential Synthetic Tool: Photocycloaddition. Chemical Reviews, 116, 9914-9993. https://doi.org/10.1021/acs.chemrev.6b00040

[10] Maddila, S., Jonnalagadda, S.B., Gangu, K.K. and Maddila, S.N. (2017) Recent Advances in the Synthesis of Pyrazole Derivatives Using Multicomponent Reactions. Current Organic Synthesis, 14, 634-653. https://doi.org/10.2174/1570179414666161208164731

[11] Lupacchini, M., Mascitti, A., Giachi, G., Tonucci, L., d'Alessandro, N., Martinez, J. and Colacino, E. (2017) Sonochemistry in Non-Conventional, Green Solvents or Solvent-Free Reactions. Tetrahedron, 73, 609-653. https://doi.org/10.1016/j.tet.2016.12.014

[12] Suzuki, S., Sakaki, S., Ishizuka, S., Nishino, T., Ito, H., Nonaka, R., Noike, M., Kodama, T., Nozaka, H., Sato, T., Agematsu, H., Maruyama, K., Oyamada, S., Kuroishi, T., Sasaki, K., Yagawa, K., Yoshioka, M. and Yokoyama, Y. (2018) Efficient Solvent- and Catalyst-Free Syntheses of Imine Derivatives Applying the Pressure Reduction Technique: Remarkable Change of the Reaction Rate with the Phase Transition. Green and Sustainable Chemistry, 8, 167-179.

[13] Ruiz-Castaneda, M., Carrion, M.C., Santos, L., Manzano, B.R., Espino, G. and Jalon, F.A. (2018) A Biphasic Medium Slows Down the Transfer Hydrogenation and Allows a Selective Catalytic Deuterium Labeling of Amines from Imines Mediated by a Ru-H/D ${ }^{+}$Exchange in D2O. ChemCatChem, 10, 5541-5550. https://doi.org/10.1002/cctc.201801343

[14] Dekamin, M.G., Azimoshan, M. and Ramezani, L. (2013) Chitosan: A Highly Efficient Renewable and Recoverable Bio-Polymer Catalyst for the Expeditious Synthesis of $\alpha$-Amino Nitriles and Imines under Mild Conditions. Green Chemistry, 15, 811-820. https://doi.org/10.1039/c3gc36901c

[15] Ghafuri, H. and Roshani, M. (2014) Aqueous Formic Acid: An Efficient, Inexpensive and Environmentally Friendly Organocatalyst for Three-Component Strecker Synthesis of $\alpha$-Aminonitriles and Imines with Excellent Yields. RSC Advances, 4, 58280-58286. https://doi.org/10.1039/C4RA11957F

[16] Jaiswal, G., Landge, V.G., Jagadeesan, D. and Balaraman, E. (2016) Sustainable Iron-Catalyzed Direct Imine Formation by Acceptorless Dehydrogenative Coupling of Alcohols with Amines. Green Chemistry, 18, 3232-3238. https://doi.org/10.1039/C6GC00565A

[17] Wermann, K., Walther, M., Goerls, H. and Anders, E. (2003) Bis(1,3,4-thiadiazolo)1,3,5-triazinium Halides: Access to Highly Substituted Aromatic Guanidines. Synlett, 2003, 1459-1462. https://doi.org/10.1055/s-2003-40844

[18] Neuvonen, H., Neuvonen, K. and Fueloep, F. (2006) Substituent Cross-Interaction Effects on the Electronic Character of the $\mathrm{C}=\mathrm{N}$ Bridging Group in Substituted Benzylidene Anilines-Models for Molecular Cores of Mesogenic Compounds. A 13C NMR Study and Comparison with Theoretical Results. The Journal of Organic Chemistry, 71, 3141-3148. https://doi.org/10.1021/jo0600508

[19] Karki, S.S., Butle, S.R., Shaikh, R.M., Zubaidha, P.K., Pedgaonkar, G.S., Shendarkar, G.S. and Rajput, C.G. (2010) Synthesis and Biological Evaluation of Some Novel Substituted $\mathrm{N}$-benzylideneaniline Derivatives. Research Journal of Pharmaceutical, Biological and Chemical Sciences, 1, 707-717.

[20] Elmakssoudi, A., Zahouily, M., Mezdar, A., Rayadh, A. and Sebti, S. (2005) $\mathrm{Na}_{2} \mathrm{CaP}_{2} \mathrm{O}_{7}$ a New Catalyst for the Synthesis of $\alpha$-Amino Phosphonates under Sol- 
vent-Free Conditions at Room Temperature. Comptes Rendus Chimie, 8, 1954-1959. https://doi.org/10.1016/j.crci.2005.05.006

[21] Gnana kumar, G., Kirubaharan, C.J., Rhan Kim, A. and Yoo, D.J. (2012) Catalytic Activity of Green and Recyclable Nanometric Tin Oxide-Doped Silica Nanospheres in the Synthesis of Imines. Industrial \& Engineering Chemistry Research, 51, 15626-15632. https://doi.org/10.1021/ie302301p

[22] He, R., Jin, X., Chen, H., Huang, Z.-T., Zheng, Q.-Y. and Wang, C. (2014) Mn-Catalyzed Three-Component Reactions of Imines/Nitriles, Grignard Reagents, and Tetrahydrofuran: An Expedient Access to 1,5-Amino/Keto Alcohols. Journal of the American Chemical Society, 136, 6558-6561. https://doi.org/10.1021/ja503520t

[23] Rai, M., Khera, V., Kaul, V.K. and Sharma, J.R. (2006) Schiff Bases of Toluidines: Synthesis and Effect of Position of Methyl Group on Biological Potential. Journal of the Indian Chemical Society, 83, 208-209.

[24] Li, B., Bheeter, C.B., Darcel, C. and Dixneuf, P.H. (2011) Sequential Catalysis for the Production of Sterically Hindered Amines: Ru(II)-Catalyzed C-H Bond Activation and Hydrosilylation of Imines. ACS Catalysis, 1, 1221-1224.

https://doi.org/10.1021/cs200331m

[25] Jiang, L., Jin, L., Tian, H., Yuan, X., Yu, X. and Xu, Q. (2011) Direct and Mild Palladium-Catalyzed Aerobic Oxidative Synthesis of Imines from Alcohols and Amines under Ambient Conditions. Chemical Communications, 47, 10833-10835. https://doi.org/10.1039/c1cc14242a

[26] Hosseini-Sarvari, M. (2011) Nanotube $\mathrm{TiO}_{2}$ as a New Catalyst for Eco-Friendly Synthesis of Imines in Sunlight. Chinese Chemical Letters, 22, 547-550. https://doi.org/10.1016/j.cclet.2010.11.017

[27] Mamedov, V.A., Mamedova, V.L., Kadyrova, S.F., Galimullina, V.R., Khikmatova, G.Z., Korshin, D.E., Gubaidullin, A.T., Krivolapov, D.B., Rizvanov, I.K., Bazanova, O.B., Sinyashin, O.G. and Latypov, S.K. (2018) Synthesis of 3-Hydroxy-4-arylquinolin2-ones Including Viridicatol via a Darzens Condensation/Friedel-Crafts Alkylation Strategy. The Journal of Organic Chemistry, 83, 13132-13145. https://doi.org/10.1021/acs.joc.8b01871

[28] Kim, M., Knettle, B.W., Dahlen, A., Hilmersson, G. and Flowers, R.A. (2003) Reduction and Reductive Coupling of Imines by Sm(II)-Based Reagents. Tetrahedron, 59, 10397-10402. https://doi.org/10.1016/j.tet.2003.06.004

[29] Gaeta, C., Talotta, C. and Neri, P. (2014) Pseudorotaxane Orientational Stereoisomerism Driven by $\pi$-Electron Density. Chemical Communications, 50, 9917-9920. https://doi.org/10.1039/C4CC04668D

[30] Lee, O.-Y., Law, K.-L. and Yang, D. (2009) Secondary Amine Formation from Reductive Amination of Carbonyl Compounds Promoted by Lewis Acid Using the $\mathrm{InCl}_{3} / \mathrm{Et}_{3} \mathrm{SiH}$ System. Organic Letters, 11, 3302-3305.

https://doi.org/10.1021/ol901111g

[31] Yang, Z., Chen, N. and Xu, J. (2015) Substituent-Controlled Annuloselectivity and Stereoselectivity in the Sulfa-Staudinger Cycloadditions. The Journal of Organic Chemistry, 80, 3611-3620. https://doi.org/10.1021/acs.joc.5b00312

[32] William, T.B. and Mohammad, M.D. (1991) Cycloaddition of ( $N$-alkyl- $N$-phenylamino) ketene with Imines. The Journal of Organic Chemistry, 56, 6118-6122. https://doi.org/10.1021/jo00021a029

[33] Lee, B., Lee, K.H., Lim, B.W., Cho, J., Nam, W. and Hur, N.H. (2013) Direct Synthesis of Imines via Solid State Reactions of Carbamates with Aldehydes. Advanced Synthesis \& Catalysis, 355, 389-394. https://doi.org/10.1002/adsc.201200907 
[34] Okamoto, S., Ariki, R., Tsujioka, H. and Sudo, A. (2017) A Metal-Free Approach to 1,2-Diamines via Visible Light-Driven Reductive Coupling of Imines with Perylene as a Photoredox Catalyst. The Journal of Organic Chemistry, 82, 9731-9736. https://doi.org/10.1021/acs.joc.7b01838

[35] Han, L., Xing, P. and Jiang, B. (2014) Selective Aerobic Oxidation of Alcohols to Aldehydes, Carboxylic Acids, and Imines Catalyzed by a Ag-NHC Complex. Organic Letters, 16, 3428-3431. https://doi.org/10.1021/ol501353q

[36] Schaufelberger, F., Hu, L. and Ramstroem, O. (2015) trans-Symmetric Dynamic Covalent Systems: Connected Transamination and Transimination Reactions. Chemistry-A European Journal, 21, 9776-9783.

https://doi.org/10.1002/chem.201500520 